\title{
ELAVANCE DE LA HISTORIA Y LAARQUEOLOGÍA DEL ORIENTE PRÓXIMO Y MEDIO EN LA CIENCIA ESPAÑOLA EN EL CAMBIO DE SIGLO (1997-2018)
}

\author{
Carmen del Cerro Linares \\ (Universidad Autónoma de Madrid)
}

\section{RESUMEN}

Esta contribución pretende hacer balance y reflexionar sobre la Ciencia en Oriente Próximo y Medio durante las dos últimas décadas en España. Nuestra participación pretende enfatizar la presencia de los estudios del Oriente Próximo y Medio en las universidades y centros de investigación españoles, así como su desarrollo en estas dos décadas.

\section{PALABRAS CLAVE}

Ciencia española en Oriente, Estudios del Próximo Oriente en España, excavaciones arqueológicas españolas en Oriente.

\section{ABSTRACT}

This contribution aims to strike Balance and think over the Near and Middle East Science during the last two decades in Spain. Our participation only intends to emphasize the presence of Studies of the Near and Middle East in Spanish Universities and Research Centres, as well as their development in these two decades

\section{KEYWORDS}

Spanish Science on Near East, Near Eastern studies in Spain, Spanish Archaeological excavations on Near East

\section{Introducción}

En 1996, Joaquín Ma Córdoba Zoilo (a la sazón profesor titular de departamento de Historia Antigua, Historia Medieval y Paleografía y Diplomática de la Universidad Autónoma de Madrid) organizó el primer congreso internacional sobre Oriente en nuestra universidad. Aquel Ier Symposium Internacional. Una década de estudios sobre el Oriente antiguo (1986-1996). Perspectivas, desarrollo y lineas de Investigación, tuvo lugar en la Facultad de Filosofía y Letras de la UAM, los días 2-4 de diciembre. En realidad, como su nombre indicaba, el simposio ya cerraba una década de estudios reflejados en lo que se había dado en llamar Ciclos de estudio sobre el Oriente Antiguo (1986-1996) en los que J. M ${ }^{\mathrm{a}}$. Córdoba ya aportaba su experiencia directa a la Ciencia española a través de su estancia como investigador en el Museo Nacional de Iraq en 1982, las prospecciones y estudio del material del valle del Balih en 1986 y 1987 o sus recién iniciadas excavaciones en el emirato de Sharjah en 1994, a la vez que preparaba su cercana vuelta a Iraq, esta vez para comenzar a excavar en Tell Mahuz.

Volviendo al Ier Symposium Internacional, aunque nuestra ciencia no estaba en sus inicios, no creo que España hubiera reunido hasta ese momento tal cantidad de investigadores europeos: H. Klengel, S. Mazzoni, F. Baffi, S. Cleuziou, M. Mouton, M. Liverani, M. Frangipane, J. Perrot, M. Salvini, R. Boucharlat, K. Kessler, E. Klengel Brandt, P. Matthiae, A. Archi, G. Bigga, H. de Meulenaere y R. Tefnin, junto a algunos españoles como J. L. Cunchillos, J. Ma Córdoba, J. Sanmartín, F. Lara, J. Ga Recio, R. Jiménez, M. Molina, C. Pérez Die, C. Sevilla, A. Morales, A. González, H. Sanz, M. Ga Antón, M. 
Pozo, T. Fdez. Pareja, M. Farjas y A. Llanos ${ }^{1}$. Ahora que lo pienso, la inmensa mayoría de los investigadores de los que se hablaba en mi programa de Historia de Oriente de cuarto curso (única asignatura de aquella antigua licenciatura relativa al Oriente Próximo y Medio que se podía cursar) impartida por el Prof. J. Ma Córdoba estaban allí. Me pareció asombroso y más aún formar parte del Symposium, colaborando en la organización del mismo.

Suponían las intervenciones de los orientalistas allí congregados, y sobre todo su presencia, un espaldarazo a lo que quedaba por venir, algo más de dos décadas de Estudios Orientales que han llegado a situar a la Ciencia española entre las, si no imprescindibles en el mundo del Oriente antiguo, sí en una de las más visibles dentro del panorama internacional. Nuestra ciencia, a lo largo de estos años, se ha consolidado a la vez que lo hacían y se creaban, grupos de trabajo, institutos superiores, excavaciones arqueológicas, revistas científicas, se introducían materias en las licenciaturas, grados y postgrados, las universidades ejercían de anfitrionas de los congresos más importantes y se defendían tesis doctorales. Así, y a lo largo de veinte años ${ }^{2}$, el panorama español es realmente esperanzador, a pesar de escaso apoyo institucional que recibe en la mayor parte de los casos.

Esos veintidós años se cerraron para nosotros con un nuevo encuentro el $I I$ Symposium Internacional, Viginti annis in studiis Orientis (1999-2019), celebrado el 26 de noviembre de 2018 en la Universidad autónoma de Madrid, que hacía balance, como nuestra contribución, de la proyección de la Ciencia española en el Oriente a lo largo de esta etapa que ahora rememoramos y que iremos desgranando a lo largo de nuestro estudio.

\section{Institutos, grupos de trabajo y sociedades científicas (por orden de creación):}

2.1. La Asociación Española de Orientalistas (AEO), fue fundada en 1963 por sacerdote jesuita F. Ma Pareja i Casañas y con sede particular Madrid primero y en la UAM después hasta 2009. A lo largo de su existencia ha convocado quince congresos nacionales bajo el título de Simposio de Culturas Orientales de la AEO.

2.2. El Grupo de Investigaciones Arqueológicas del Mediterráneo y Próximo Oriente (GRAMPO)/Seminario de Arqueología Prehistórica del Próximo Oriente (SAPPO) ${ }^{3}$ del departamento de Prehistoria de la Universidad Autónoma de Barcelona, fue creado en 1989. Dirigido por M. Molist Montañà, el SAPPO ha sido reconocido como Grupo de Investigación de Calidad en 2005 y 2012 y es, quizás, el equipo más reconocido internacionalmente por su presencia continua en Siria, Turquía e Iraq.

2.3. El Instituto del Próximo Oriente Antiguo (IPOA) ${ }^{4}$, de la Universidad de Barcelona, fue creado oficialmente por el Decreto de la Presidencia de la Generalidad de Cataluña del 24 de noviembre de $1993^{5}$ (refundado desde el antiguo Instituto de Estudios Orientales, $\mathrm{IEO}^{6}$ ) y, tras varios directores, a cargo de I. J. Adiego Lajara. Ha contado con un buen número de proyectos por lo que resaltamos aquí los que continúan en la actualidad ${ }^{7}$ : The International Association for Comparative Semitics; tell Qara Quzaq; A Glossary of Old Syrian, Amorite Onomastics in the Middle Euphrates and the Habur Areas during

\footnotetext{
Desgraciadamente algunos de ellos ya no nos podrían acompañar en un nuevo Simposio. Gracias eternas a S. Cleuziou, H. de Meulenaere, J.L. Cunchillos y C. Sevilla.

2 Aunque el título de nuestra participación y el resumen de la misma lo indica, queremos recordar aquí, que nuestro estudio está acotado a los años 1997-2018, los que van desde Ier Symposium Internacional. Una década de estudios sobre el Oriente antiguo (1986-1996). Perspectivas, desarrollo y líneas de Investigación al II Symposium Internacional, Viginti annis in studiis Orientis (1999-2019).

3 http://grupsderecerca.uab.cat/sappo/es

http://www.ub.edu/ipoa/Index_esp.html

https://www.ub.edu/web/ub/es/recerca_innovacio/recerca_a_la_UB/instituts/institutspropis/ipoa.html

6 J. Vidal Palomino, Historia del Instituto del Próximo Oriente Antiguo (1971-2012), 2016, pp. 21-48

7 http://www.ub.edu/ipoa/ipoaRECERCA.htm
} 
the Middle Bronze Age: the Feminine and Masculine Anthroponomy, an Anthropological and Linguistic Analysis; Publicación y edición del archivo cuneiforme oficial mesoasirio "Assur M 8" y Catalogación y estudio de los manuscritos arameo-siriacos y árabes de la Fundación Salem y de la Biblioteca Maronita de Alepo (Siria).

2.4. El Centro de Estudios del Próximo Oriente Antiguo (CEPO) ${ }^{8}$, creado en 1993 en Madrid, es una sociedad científica dedicada a promocionar el estudio y la investigación sobre el Oriente Próximo Antiguo ideado por J. L. Cunchillos Ilarri. El Centro está actualmente dirigido por J. A. Álvarez Pedrosa y ha organizado siete congresos a nivel nacional, de ellos han sido publicadas las actas de primer, tercer, cuarto y quinto encuentro9.

2.5. El Centro Superior de Estudios del Próximo Oriente y Egipto ${ }^{10}$, (antiguo $\mathrm{CEAE}^{11}$ ) creado en 1998, sito en la Universidad Autónoma de Madrid y dirigido por J. $\mathrm{M}^{\mathrm{a}}$ Córdoba Zoilo del que se deriva el Grupo de Investigación Culturas, tecnologías y medio ambiente de las sociedades del Oriente Próximo Antiguo ${ }^{12}$, reconocido como tal en 2008 y consolidado en 2018. El Centro Superior de Estudios del Próximo Oriente y Egipto ha desarrollado los siguientes proyectos que nombramos cronológicamente: el Valle del Balih, (Siria), 1986 y 1987, al Madam (Sharjah, Emiratos Árabes Unidos). Las comunidades campesinas y la cultura de los oasis durante la Edad del Hierro en la Península de Omán, desde 1994; Mitanni y los Hurritas en el Transtigris. Historia de una ciudad y su entorno desde los orígenes hasta el Periodo Sasánida (Tell Mahuz, Iraq) 1997-2003; la parte española del Proyecto científico euro-sirio en Tell Beydar, Siria, dirigido por M. Lebeau en el que equipo español trabajó en los niveles helenísticos del yacimiento ${ }^{13}$ coordinado in situ por R. Martín Galán y desde el Centro por C. Sevilla Cueva entre 2004 y 2007; Dehistán durante la Edad del Hierro. Formas de vida, creencias y entorno en una región del Asia Central, desde 2006 y El Oriente Próximo Antiguo. Redescubrimiento, historia e investigación moderna en colaboración con F. J. Villalba Ruiz de Toledo y J. M. Herrero de la Iglesia en 2006 y 2007.

Del Centro Superior de Estudios del Próximo Oriente y Egipto y del departamento de Historia antigua de la UAM depende el Aula didáctica Antonio Blanco FreijeiroLaboratorio para la Historia Antigua de Oriente Próximo y Egipto ${ }^{14}$. En palabras de su director, J. Ma Córdoba "La colección se ha reunido para que los estudiantes universitarios puedan disponer de una documentación variada sobre la cultura, la historia, las excavaciones arqueológicas pasadas y presentes o los problemas de la investigación científica más reciente" $" 15$.

2.6. El Instituto de Estudios Islámicos y del Oriente Próximo. Unidad Próximo Oriente Antiguo $^{16}$ (Cortes de Aragón, CSIC y la Universidad de Zaragoza ${ }^{17}$ ) fue fundado en 2000 y cerrado en 2011, dirigido primero por M. Borrás Gualis y después

\footnotetext{
http://cepo.es/

$9 \mathrm{http}: / /$ cepo.es/publicaciones/

$10 \mathrm{http}: / /$ www.uam.es/otroscentros/asiriologiayegipto/

11 Centro de Estudios de Asiriología y Egiptología

$12 \mathrm{http}: / /$ uam.es/UAM/Grupos-de-investigación/Ficha/1446755836600.htm?idGrupo=407\&language=es \&nombreGrupo $=$ Culturas, $\% 20$ tecnología $\% 20 y \% 20$ medio $\% 20$ ambiente $\% 20 \mathrm{de} \% 201$ as $\% 20$ sociedades $\% 20$ del\%20Oriente\%20Próximo\%20Antiguo.\&site=UniversidadAutonomaMadrid

13 R. Martín Galán, "El período helenístico: pervivencia del legado autóctono", Cuadernos del Seminario Water Andrae 7, 2004-2005, passim

14 J.Ma Córdoba Zoilo, Aula didáctica Antonio Blanco Freijeiro. Un Laboratorio para la práctica de la Historia Antigua de Oriente Próximo y Medio. Supplementa ad Isimu. Series I: Studia, Vol. 4, Madrid, 2018

15 http://www.uam.es/otroscentros/asiriologiayegipto/aula abf/aula abf.html

$16 \mathrm{http} / / /$ ieiop.unizar.es/uinvestigacion/u3/index.php

$17 \mathrm{http}$ ///bases.cortesaragon.es/bases/NDocumen.nsf/69b541b37a1f7fb0c12576d20031f70e/c7c14cb35cd9 6943c125731700263b91/\$FILE/programa\%20instituto\%20de\%20estudios\%20isl\%C3\%A1micos.pdf
} 
por J. P. Vita Barra. Centró su labor investigadora en las culturas del antiguo Oriente Próximo, con varios proyectos, mencionamos aquí los que se centran en Oriente: Automatización del proceso de interpretación de textos; Historia social y económica de Siria-Palestina en el Bronce Final y Dialectología acadia y semítica-noroccidental en el II milenio a.C. Propició la enseñanza de las lenguas del Próximo Oriente y la escritura cuneiforme.

2.7. El Instituto Bíblico y Oriental de León (IBO) ${ }^{18}$ comenzó sus actividades en el año 2003, dirigido por J. García Recio. Su sede principal, en León, fue inaugurada en 2009 y actualmente está en proceso de traslado a Cistierna, en la Montaña Oriental leonesa. Su biblioteca, con más de 30.000 volúmenes, es la más grande de España relativa el Oriente Próximo. En el trabajo de campo, el IBO comienza a tener la presencia más reciente en Oriente a través de las excavaciones de Hirbat al-Jerahiya ${ }^{19}$ (2010-2011) y Tell Bismaya $(2018)^{20}$, Iraq

2.8. El Instituto de Lenguas y Culturas del Mediterráneo y Oriente Próximo (ILC), Departamento de Estudios de Próximo Oriente Antiguo del Centro de Ciencias Humanas y Sociales del CSIC ${ }^{21}$ nació en 2007 y proviene de antiguo Instituto de Filología del CSIC, con dos grupos de investigación relativos al Oriente Próximo: Las Letras y las Ciencias en la Antigua Mesopotamia (LYCAM), dirigido por B. Böck, desde el que se editan y estudian textos sumeroacadios y Próximo Oriente Antiguo (PROA), que, dirigido actualmente por J.P. Vita Barra, estudia las lenguas y las culturas del Próximo Oriente desde el III milenio a.C. hasta la época helenística.

2.9. El Centro de Estudios del Próximo Oriente y Antigüedad Tardía (CEPOAT) ${ }^{22}$ surgió del Instituto del Próximo Oriente Antiguo (IPOA) de Barcelona y de la labor incansable del profesor A. González Blanco ${ }^{23}$, transformado en Murcia en 2011 a Centro de Estudios del Próximo Oriente y la Antigüedad Tardía. Dirigido por G. Matilla Séiquer, el CEPOAT ha trabajado Siria en las excavaciones de urgencia por la construcción de la presa de Tisrin, rescatando Tell Qara Quzaq ${ }^{24}$ (1989-1999) y Tell Jamis ${ }^{25}$ (1992-2010). La construcción de la presa se retrasó y eso permitió un estudio integral de los yacimientos, sobre todo de Tell Jamis ${ }^{26}$. Actualmente el Centro desarrolla los proyectos: Mundo y lengua Elamita $^{27}$; Epigrafía y Arquitectura en Qal'at Nadjem. Es uno de los centros más activos desde el punto de vista de la didáctica y enseñanza del Próximo Oriente, a través del Aula virtual del CEPOAT ${ }^{28}$ dirigida por G. Matilla Séiquer ${ }^{29}$.

\footnotetext{
18 https://institutobiblicoyoriental.wordpress.com/el-ibo/

19 M. García Fernández et al., "Investigación arqueológica en Hirbat al-Jerahiya (Iraq), noviembre de 2011-noviembre de 2013", Memoria académica 2012-2013, Universidad Pontifica de Comillas, Proyecto $\mathrm{I}+\mathrm{D}$, p. 290,2013

20 Agradecemos a J. García Recio (director del IBO) que nos haya hecho llegar el Informe de la visita preliminar a Tell Bismaya (Adab), que tuvo lugar el 7-8 de noviembre de 2018 y que arranca lo que esperamos que sea el primer y gran proyecto español en Mesopotamia.

$21 \mathrm{http} / / /$ ilc.csic.es/es/research-dpto/dpto-estudios-proximo-oriente-antiguo

22 https://www.um.es/cepoat/

23 A. Egea y J.J. Martínez, “¿Orientalismo en Murcia? La labor del Profesor Antonino González Blanco”, Estudios Orientales 8, 2017, pp. 9-14.

24 J. Gallardo Carrillo y G. Matilla Séiquer, “Tell Qara Qûzâq. Un excepcional descubrimiento" Revista de Arqueología 198, 1997, pp. 24-35.

25 G. Matilla Séiquer, Arqueología y antropología en el Alto Éufrates sirio: Tell Jamîs, Murcia, 2001.

26 https://www.um.es/cepoat/telljamis/

27 https://www.um.es/cepoat/elamita/

28 Los docentes de aula se han preocupado por preparar material didáctico del que destacamos: A. Egea Vivancos et al., (Coords.), Y la arqueología llegó al aula. La cultura material y el método arqueológico para la enseñanza de la historia y el patrimonio, Gijón, 2018

29 https://www.um.es/cepoat/aulavirtual/
} 
2.10. La asociación Orients, proviene de la extinta Asociación de Amigos del CEPO (AACEPO) que había nacido en 1996 para ayudar a la difusión del CEPO ${ }^{30}$.. En 2013 la Asociación es refundada y cambia de nombre; Oriens. Asociación de Estudios del Oriente Próximo, con sede en Madrid ${ }^{31}$. Orients difunde el conocimiento del Próximo Oriente y el Mediterráneo, pero también de la Antigüedad Clásica.

\section{Excavaciones arqueológicas}

El informe realizado por Instituto del Patrimonio Cultural de España en 2007 relativo a las excavaciones arqueológicas en el exterior ${ }^{32}$, indicaba que los proyectos españoles en Oriente subvencionados por el Ministerio de Cultura eran solo seis antes de 1999. Entre ese año y 2007 España llegó a tener once proyectos subvencionados en Oriente. Todos ellos habían tenido su lugar en la exposición realizada en el Museo Arqueológico Nacional, en 2006, La aventura española en Oriente (1166-2006): viajeros, museos y estudiosos en la Historia del redescubrimiento del Oriente Próximo antiguo ${ }^{33}$ que se inauguraba a la vez que tenía lugar el V International Congress on Archaeology of Ancient Near East en Madrid, de tal suerte que todo el panorama científico internacional podía sopesar por vez primera la presencia española en Oriente. Con la salida de los equipos de Siria en 2011, el país que más misiones albergaba, de nuevo las misiones se redujeron a $\operatorname{seis}^{34}$, pero la realidad es que sumando subvenciones estatales y privadas, evitando guerras y sufriendo pérdidas, nuestra Ciencia ha llegado a estar presente en más de treinta puntos del Próximo y Medio Oriente ${ }^{35}$, a saber: Emiratos Árabes Unidos, al Madam (Universidad Autónoma de Madrid). Iraq, tell Mahuz (Universidad Autónoma de Madrid), tell Lashkry, Arbil, y Banahilk (Universidad Autónoma de Barcelona), Hirbat al-Jerahiya y tell Bismaya (Instituto Bíblico y Oriental, León). Israel, tell Hatzor (equipo hispano-hebreo de la Universidad Complutense de Madrid) y Nahal Efe (CSIC-Barcelona/Israel Antiquities Authority). Jordania, yebel Mutawwaq (misión hispano-italiana de la Universidad de Oviedo/Pontificia de Salamanca); Ciudadela de Amman y qarst Hallabat (AECID), Kharaysin-Zarqa (CSIC-Barcelona y Universidad de Cantabria) y Sela (Universidad de Barcelona). Líbano, Tiro (necrópolis y puerto, Universidad Pompeu Fabra) y tell Labwe, Beqaa (CSIC-Barcelona). Pakistán, Bhando Qubo (Universidad Pompeu Fabra). Palestina, tell el Far-Ah y Khirbet Rabud (Universidad de la Coruña). Siria, tell Halula y Chagar Bazar (Universidad Autónoma de Barcelona); Monasterios del Éufrates, tell Jamis, tell Qara Quzaq y qal'at Nadjem (Universidad de Murcia); tell Beydar, (equipo euro-sirio de la Universidad Autónoma de Madrid); Halabiyé, tell Humeida (misión sirio-española) y tell Qubr Abu al-'Atiq (Universidad de la Coruña). Turkmenistán, Geoktchik depe e Izat Kulli (Universidad Autónoma de Madrid). Turquía, Tilbes hüyük, (misión turco-española de la Universidad de Alicante) y Akarçay tepe (misión turco-española de la Universidad

\footnotetext{
30 Vid supra, p.4

$31 \mathrm{http}: / /$ oriens.es/oriens/node/3

32 C. Martín, Las ayudas a proyectos de investigación arqueológica en el exterior de la dirección general de bellas artes y bienes culturales del ministerio de cultura. Instituto del Patrimonio Cultural de España, 2007, passim.

33 La publicación de la exposición supuso la aparición de dos volúmenes, el segundo La aventura española en oriente (1166-2006): viajeros, museos y estudiosos en la Historia del redescubrimiento del Oriente Próximo antiguo; la arqueología española en Oriente: nacimiento y desarrollo, editado por sus comisarios, J. Ma . Córdoba y C. Pérez Díe en Madrid, 2006, daba cuenta de todas y cada una de estas intervenciones arqueológicas.

34 Podemos contar las excavaciones abiertas en la publicación del MCU Informes y Trabajos Excavaciones en el exterior 9, 2011.

35 No incluimos aquí a la cantidad de colegas españoles diseminados por el Oriente Próximo y Medio que trabajan para misiones sin dirección española o que dirigen excavaciones, pero anclados en instituciones que no son españolas, como es el caso de Tell Regev (Israel) dirigido desde la Saint Louis University, Madrid.
} 
Autónoma de Barcelona). Uzbekistán, Termez (Universidad de Barcelona). Yemen, Zafar (Universidad Autónoma de Barcelona).

\section{Revistas científicas}

La creación de institutos y sus correspondientes proyectos ha fomentado el nacimiento de revistas científicas relacionadas con nuestra Ciencia, mencionaremos aquí las de mayor reconocimiento:

4.1 El Boletín de la Asociación Española de Orientalitas fue editado desde la AEO con un número anual desde 1965 a 2009, se editaba en Madrid y contaba con el mecenazgo de la Agencia Española de Cooperación Internacional.

4.2 La revista Aula Orientalis. Revista de estudios del Próximo Oriente Antiguo ${ }^{36}$ se publica desde 1983 en Sabadell y está, dirigida por G. del Olmo Lete, actualmente profesor emérito de la Universidad de Barcelona, cuenta además con el asesoramiento cientifico del IPOA (UB)

4.3. La revista Estudios Orientales ${ }^{37}$ fue fundada por A. GonzalezBlanco (Universidad de Murcia/CEPOAT), que editó su primer volumen en 1997. Actualmente está dirigida por A. Egea Vivancos. En 2017 editó su octavo número dedicado a su fundador.

4.4 La revista Isimu. Revista sobre Oriente Próximo y Egipto en la Antigüedad ${ }^{38}$ es editada por el Centro de Estudios de Oriente Próximo y Egipto desde 1998, con carácter anual y está dirigida por J. $M^{a}$ Córdoba Zoilo. La Publicación se encuentra reforzada por los llamados Suplementa ad Isimu repartidos en cinco series: I Series: Studia, II Series: Acta et Symposia, III Series: Varia, IV Series: Colloquia y V Series: Studia in itinere.

\section{Materias en licenciaturas, grados y másteres}

Pero toda ciencia que se precie debe acercarse a las aulas. Desgraciadamente no todos los equipos ni todas las universidades e institutos lo han conseguido, realidad que dificulta enormemente la transmisión del saber Próximo y Medio Oriental, a aquellos que en un futuro podrían seguir nuestro camino. Es cierto que, como materia básica de carácter general y bajo el nombres como: Historia de Oriente Próximo en la Antiguedad, Historia Antigua del Próximo Oriente, Sociedades y Culturas del Próximo Oriente Antiguo, Historia y Civilización del Próximo Oriente Antiguo, Prehistoria e Historia Antigua de Oriente, Arqueología del Próximo Oriente, Civilizaciones de Egipto y el Próximo Oriente o Historia del Oriente Próximo y Egipto, nuestra materia (compartiendo la mayor parte de las veces programa con el antiguo Egipto) ya forma parte de los Grados de Historia y Ciencias de la Antigüedad de las universidades de Barcelona, Autónoma de Barcelona, Sevilla, la Coruña, Alicante, Complutense de Madrid y Autónoma de Madrid, entre otras.

Lo que ya es más difícil es que esa enseñanza (histórica, arqueológica o filológica) supere el nivel básico y forme parte del plan de estudios en asignaturas de especialidad y de postgrado. Aun así, contamos ciertas posibilidades en el panorama español:

5.1. San Dámaso. Facultad de Literatura Cristiana y Clásica San Justino ${ }^{39}$. La Facultad ofrece la posibilidad de estudiar varios cursos de lenguas (libre acceso) en: Hebreo, Armenio, Arameo, Siríaco, Acadio, Árabe, Sumerio, Persa (nivel inicial, medio y avanzado)

5.2. CEPOAT. Cursos on line: Acadio, Elamita, Hitita, Sumerio, Hebreo y Arameo (nivel inicial, medio y avanzado), Arte hitita, Historia hitita, Arqueología fenicia, Arqueología púnica y Arte de Babilonia y Asiria.

\footnotetext{
36 http://www.ub.edu/ipoa/

$37 \mathrm{https}: / /$ www.um.es/cepoat/estudiosorientales/?page_id=131

$38 \mathrm{https}: / /$ revistas.uam.es/isimu

$39 \mathrm{https}: / / \mathrm{www} \cdot$ sandamaso.es/literatura
} 
5.3. IBO. Repartido entre sus sedes de León, Cistierna, Valladolid, Madrid, Santiago de Compostela, Vitoria-Gasteiz y San Antonio (Texas) el instituto imparte cursos de acadio, sumerio y religión mesopotámica.

5.4 UAB, Facultad de Filología. Departamento de Estudios Hebreos y Arameos Incluye dentro del Máster de Culturas y Lenguas de la Antigüedad, coordinado por E. E. Marcos Hierro, la Especialidad B: Biblia y Oriente Próximo Antiguo ${ }^{40}$. Las asignaturas relacionadas con Oriente son: Introducción a la Historia de las Culturas del Mediterráneo y del Próximo Oriente Antiguo, Lengua Acadia, Lengua Sumeria I y II, Lengua Ugarítica, Lengua y Cultura Arameas: desde el $S$. X a.C. hasta el presente, Lenguas Indoeuropeas Orientales, Sistemas de Escritura del Mediterráneo y del Próximo Oriente Antiguo, Sistemas Literarios del Mediterráneo y del Próximo Oriente y Religiones del Próximo Oriente Antiguo.

5.5. UAB/IPOA. El instituto ofrece un Máster en Asiriología ${ }^{41}$, de corte básicamente filológico, que en su edición de 2017-18, permitía cursar: Lengua acadia I y II, Lengua sumeria I, II y III, Cultura mesopotámica, Textos acadios epistolares y administrativos paleobabilónicos y Textos literarios de tradición babilónica.

5.6. UAM. Facultad de Filosofía y Letras. Grado de Historia. Ofrece asignaturas de especialidad además de las básicas: Fenicios, griegos y cartagineses en el Mediterráneo, Historia de Oriente en el II milenio: de los amorreos a los grandes reyes y Del Oriente Romano a Bizancio.

5.7. UAM. Facultad de Filosofía y Letras. Grado de Ciencias y Lenguas de la Antigüedad y Doble grado en Historia del Arte y Ciencias y Lenguas de la Antigüedad. Ofrece asignaturas de especialidad además de las básicas: Introducción a las lenguas antiguas del Próximo Oriente (Sumerio y acadio), Historia de oriente: del imperio asirio a los imperios iranios y Arqueología de Oriente y Egipto. Este es el único caso en el que las lenguas del Próximo Oriente se imparten en un Grado oficial.

5.8. UAM-UCM. Máster Interuniversitario en Historia y Ciencias de la Antigüedad ${ }^{42}$ coordinado por E. Sánchez Medina y F. Echeverría Rey y que en su módulo 2 oferta la Especialidad de Oriente y Egipto antiguos. Las materias impartidas entre las dos universidades son: Estados e imperios en el Oriente antiguo, Babilonia: historia y cultura, Asiria: historia y cultura, Acadio, Legado y redescubrimiento de la Antigüedad; El redescubrimiento de Oriente y La transición del mundo antiguo al mundo medieval; Oriente romano y bizantino.

5.9 UAM/CEAE. El Centro Superior de Oriente y Egipto realiza Cursos de Formación continua, solo mencionaremos aquí los que se imparten en el curso 2018-19: El III milenio a.C. en Oriente Próximo (sumerios y acadios): Hechos, espacios y lenguas y El III milenio a.C. en Oriente Próximo (Asia Central, Siria y Anatolia): Ejes, materias y lenguas ${ }^{43}$.

5.10. UAH dentro del Grado de Historia se oferta una asignatura de especialidad, Las mujeres en el Próximo Oriente antiguo.

5.11. UAH. Cursos de Extensión Universitaria. Cursos en Acadio, Sumerio y un curso sobre el enuma elish ${ }^{44}$

\footnotetext{
$40 \mathrm{http} / /$ www.ub.edu/estudis/es/mastersuniversitaris/cla/plan-de-estudios

41 http://www.ub.edu/ipoa/ACAD-ESP-ASIRIOLOGIA.htm

${ }^{42} \mathrm{http}: / /$ www.uam.es/ss/Satellite?c=Page\&cid=1446766291534\&language=es\&nodepath=Plan+de+estudi os\&pagename $=$ FacdFilosofia $\% 2$ FPage $\% 2$ FUAM contenidoFinal

43 https://uam.es/ss/Satellite/es/1242652866332/1242700425487/cursocortaduracion/cursoCortaDuracion/ El_III_milenio_a._C._en_Oriente_Proximo_(sumerios_y_acadios):_Ejes,_materias_y_lenguas_ $(4 \% \mathrm{C} 2 \% \mathrm{AA}$ edicion $) \cdot \mathrm{htm}$

$44 \mathrm{http}: / / w w w 3$. uah.es/cultura/index.php/home/categoryevents/13?start=30
} 
5.12. UOC. Master Interuniversitario Mediterráneo Antiguo (UOC, UAH y UAB), coordinado por G. Munilla Cabrillana, I. B. Antela-Bernárdez y F. J. Gómez Espelosín, con Especialidad en Egipto y Próximo Oriente, donde se imparte ${ }^{45}$ : Retórica del poder e imperios mesopotámicos e Historia cultural del Próximo Oriente y el Antiguo Egipto.

\section{Congresos}

El número de especialistas que ya se daban cita en nuestra aulas e institutos, ha permitido que nuestra Ciencia sea la anfitriona en tres eventos de gran calado. Así, la Universidad Autónoma de Madrid albergó el primer gran encuentro científico internacional, al ser la sede del V International Congress on the Archeology of the Ancient Near East, celebrado entre el 3 y 8 abril de 2006. El año 2010 fue sin embargo significativo para Barcelona, ya que el Grupo de Investigaciones Arqueológicas del Mediterráneo y Próximo Oriente (GRAMPO-UAB) convocó la tercera edición del Broadening Horizons. Conference of young Researchers working in the Ancient ${ }^{46}$, encuentro que con su sexta edición (Berlín, junio de 2019) se ha convertido en todo un referente para los jóvenes orientalistas europeos. Además, esta ciudad albergó el 56 Rencontre Assyriologique Internationale, entre el 26 y el 30 de julio. Las actas de los tres congresos han sido editadas por sus respectivos comités ${ }^{47}$.

\section{Compendio de tesis doctorales $(1997-2018)^{48}$}

Fructuoso Martínez, Pedro, Urartu. Universidad de Murcia.

García Trabazo, José Virgilio, Los textos hititas en "Ancient Near Eastern Texts relating to the Old Testament” (ANET): edición critica y traducción. Universidad de Oviedo.

Matilla Séiquer, Gonzalo, Arqueología y antropología en el alto Éufrates Sirio: Tell Jamis. Universidad de Murcia.

Zamora López, José Ángel, La vid y el vino en Ugarit. Universidad de Zaragoza.

2000

Feliu Mateu, Lluís, El deu Dagan a la Siria de l'edat del Bronze Universidad de Barcelona.

González Salazar, Juan Manuel, La política y administración de las fronteras del Reino Hitita. Estudio comparativo del territorio limitrofe del sector septentrional de Anatolia durante gran parte del II milenio A.C. Universidad Autónoma de Madrid.

\footnotetext{
45 https://estudios.uoc.edu/documents/12253/1261065/MS31-ES-MUMA-AIH.pdf/b398afb9-cf5a-4668$868 \mathrm{c}-40 \mathrm{e} 347 \mathrm{~d} 1 \mathrm{baf0}$

46 Las actas del BH3 fueron publicadas por F. Borrell Tena et al., Broadening horizons 3. Conference of Young Researchers working in the Ancient Near East, Barcelona, 2012.

47 J. Córdoba Zoilo et al. (Coord.), Proceedings of the 5th International Congress on the Archaeology of the Ancient Near East, Madrid, 2008; L. Feliu et al. Time and History in the Ancient Near East, Winona Lake, 2013

48 Por falta de espacio, vamos a mencionar en este apartado solo las tesis presentadas que tengan un marcado acento histórico, dejando a un lado las puramente filológicas o las que cronológicamente se inserten en el Paleolítico o la época islámica. Hemos realizado este listado con las tesis incluidas en las bases de datos (la mayor parte de ellas actualizadas solo hasta 2017) o con las que tenemos constancia sin excluir premeditadamente ninguna. Pedimos disculpas ante alguna omisión no deliberada.
} 
Llop Raduà, Jaume, Aportació a l'estudi de les relacions polítiques i militars entre Assíria i Babilònia durant la segona meitat del segon mil.leni a.C. Universidad de Barcelona.

2004

Cerro Linares, Carmen del, Espacio arquitectónico y sociedad durante la Edad de Hierro en la Península de Omán (1300-300 a.C.) Universidad Autónoma de Madrid.

Martín Galán, Rodrigo, Asentamientos de época Seleuco-Parta en la Alta Mesopotamia. Universidad Autónoma de Madrid.

Río Alda, Ángel del, Escritura y alfabetización. Su impacto en la Antigüedad. Universidad Complutense de Madrid.

Vidal Palomino, Jordi, Las aldeas de Ugarit según los archivos del Bronce Reciente (ss. XIV-XII a.n.e.) Universidad Autónoma de Barcelona.

2005

Cruells, Walter, Orígens, emergència $i$ desenvolupament de la ceràmica Halaf a Síria. Universidad Autónoma de Barcelona.

Egea Vivancos, Alejandro, Poblamiento romano en el alto Éufrates sirio. Universidad de Murcia.

Guadalupe Ingelmo, Salomé, La divina serpiente. Estudio sobre las divinidades ofídicas mesopotámicas. Universidad Autónoma de Madrid.

2006

Akram Hamouri, Khaled, La religión de los nabateos. Universidad Complutense de Madrid.

Guerrero Vila, Emma, Estudio demográfico de la población en el Neolítico de Próximo Oriente. El caso de Tell Halula (valle del Éufrates, Siria) y su contextualización en el Levante mediterráneo. Universidad Autónoma de Barcelona.

2007

Justel Vicente, Josué Javier, La posición social de la mujer en la Siria del Bronce Final. Universidad de Zaragoza.

2008

Núñez Calvo, Francisco Jesús, Estudio cronológico-secuencial de los materiales cerámicos de la necrópolis fenicia de Tiro-Al Bass (Líbano). Universidad Pompeu Fabra.

2009

Escribano Martín, Fernando Creencias religiosas, fiesta y espacio urbano en la Babilonia caldea. Universidad Autónoma de Madrid / Università degli Studi di Roma la Sapienza.

2010

Velázquez Muñoz, Joaquín, El sistema de caminos reales en el imperio persa aqueménida. Universidad Complutense de Madrid.

2011

Gómez Bach, Anna, Caracterización del producto cerámico en las comunidades neolíticas de mediados del V milenio Cal BC: El valle del Éufrates y el valle del Khabur en el Halaf final (Siria). Universidad Autónoma de Barcelona. 
Soláns Gracia, Bárbara Eugenia, Poderes colectivos en la Siria del Bronce Final. Universidad de Zaragoza.

2012

Al Khabour, Anas, Historia de la ocupación del valle del Éufrates entre el Balih y el Habur hasta la conquista de Alejandro. Universidad Autónoma de Madrid/ Centro Superior de Investigaciones Científicas (Centro de Ciencias Humanas y Sociales), Madrid.

Bouso García, Mónica, Las Prácticas funerarias en el Valle del Éufrates durante el tercer milenio y la primera mitad del segundo: Estudio intertextual a partir de las evidencias arqueológicas y epigráficas. Universidad de Barcelona.

Justel Vicente, Daniel, Aspectos legales de la infancia en el Próximo Oriente antiguo durante el Bronce Reciente (CA. 1500-1100 A. C.) Universidad de Zaragoza.

Sánchez Ruiz, Cruz, Vallée Du Khabour. Quartiers d'Habitation et premiers moments de l'urbanisme en Mesopotamie du nord. Universidad Pompeu Fabra.

Torrecilla Giménez, Eduardo, Late Bronze Age Ekalte. Chronology, Society, and Religion of Town in the Land of Aštata. Universidad de Castilla-La Mancha.

2013

Gil Fuensanta, Jesús, La arquitectura de Anatolia oriental durante el IV milenio A.C. en el marco del urbanismo del Próximo Oriente y Egipto. Universidad Autónoma de Madrid.

2014

Arroyo Cambronero, Ana, El agua dulce en la cultura hitita. Universidad Autónoma de Madrid / Freie Universität Berlin.

Hernández Álvarez, Jorge, Estudio de Isin en el III milenio a través de sus fuentes arqueológicas y textuales. Universidad Complutense de Madrid / Universidad Autónoma de Madrid.

Ortiz López, Anabel, Estudio arqueo-antropológico de las sepulturas del PPNB Medio y Reciente del yacimiento de Tell Halula (Valle Medio del Éufrates, Siria). Universidad Autónoma de Barcelona.

2015

Marchiori, Chiara, Arquitectura en tierra de la prehistoria y protohistoria en el Próximo Oriente. Estudio arqueométrico del adobe en los yacimientos de Tell Halula, Yumuktepe y Tell Tuqan, Universidad Autónoma de Barcelona.

2016

Faura, Josep-Miquel, El conjunt ceràmic del VII mil·lenni Cal bc de tell halula (Síria). Una evidència dels processos de canvi socioeconòmic. Universidad Autónoma de Barcelona.

Pintado Martínez-Meco, Manuel, Relaciones internacionales en SiriaMesopotamia (ca. 2400-2300 a.N.E.). La carta de Enna-dagan y el tratado entre Ebla y Abarsal. Universidad de Castilla la Mancha.

Rodríguez Marco, Gabriel, La diosa Inanna y Nippur: la Hierogamia y el viaje al inframundo. Una investigación interdisciplinar. Universidad Complutense de Madrid.

Sánchez Priego, Juan Antonio, Producción y uso de azuelas, hachas y martillos en el neolítico precerámico de Siria ( $X^{o}$-VII ${ }^{o}$ milenios cal. a.C.) Aportes de la tecnología y la experimentación al estudio de la neolitización del Levante. Universidad Autónoma de Barcelona. 
Sanchiz Álvarez de Toledo, Hipólito, Testimonios históricos y míticos de la ciudad Suruppak. Universidad Complutense de Madrid.

Taha, Buchra, Industria ósea en el Neolítico del Próximo Oriente. Estudio tecnológico y funcional del asentamiento de Tell Haula (Valle del Éufrates, Siria). Universidad Autónoma de Barcelona.

2017

Alesso, Marcelo José, Los filisteos: la emigración de un pueblo y su instalación en Canaán. Universidad Carlos III.

Espejel Arroyo, Fernando, El movimiento formativo protoestatal en al Yazira y Anatolia oriental (V-IV milenios a.C.) Los procesos originales y los efectos del contacto con la I urbanización mesopotámica. Universidad Autónoma de Madrid.

González Moratinos, Sara, Antropología del parentesco en Babilonia. Estudio de los grupos consanguíneos y residenciales en el periodo paleobabilónico. Universidad de Barcelona.

Olaya Montero, Núria, Las Fuentes clásicas y orientales relativas a las fronteras septentrionales del Imperio Sasánida (224-651). Universidad Autónoma de Barcelona.

2018

Blesa Cuenca, Jose Luis, Los arios. Historia y modos de vida de los pueblos centroasiáticos de la Edad del Hierro. Universidad Autónoma de Madrid.

\section{A modo de cierre.}

Hace solo diez años, mientras preparábamos el V ICAANE, un miembro del Comité internacional nos decía que no estaba seguro de que la elección recayera en España, un "país exótico" para el Orientalismo europeo, nosotros sonreímos convencidos de que eso iba a cambiar, y así ha sido.

En veinte años, la Ciencia española dedicada al Próximo Oriente antiguo ya no es un elemento exótico. Los profesores, institutos o grupos de investigación diseminados por las universidades de Madrid, Barcelona, Murcia, la Coruña, Oviedo, Alcalá etc, así lo avalan. La mayoría están dentro de equipos interdisciplinares y en contacto directo con otros países europeos y con los próximo y medio orientales. Las más de cuarenta tesis defendidas por toda España son un ejemplo más de esta tendencia, así como la introducción de materias básicas en casi todos los grados de Historia y Ciencias de la Antigüedad, y de materias específicas en especialidad y en postgrados oficiales. España ha sido también elegida como sede de congresos, ha creado sus primeras colecciones como la del Museo de Monserrat o el IBO de León y ha remodelado sus museos dando espacio al Oriente Antiguo o aceptando grandes exposiciones como La aventura española en Oriente (1166-2006): viajeros, museos y estudiosos en la Historia del redescubrimiento del Oriente Próximo antiguo (MAN, 2006) o En los confines del Oriente Próximo. El hallazgo moderno del país de Magán. Veinte años de descubrimientos del Departamento de Antigüedades de Sharjah (Emiratos Árabes Unidos), la comunidad científica internacional y la misión española $(\mathrm{MAN}, 2016)^{49}$, sin menospreciar las más pequeñas, pero de gran importancia para que este Oriente se introduzca por todas partes en España, como la muestra titulada Ex Oriente Lux. Virgilio Sevillano, diplomacia y antigüedades, que inauguró el Museo de Zamora a finales de junio de 2019.

49 J.M Córdoba (Dir.), 2016, En los Confines de Oriente Próximo: El Hallazgo moderno del país de Magán, Madrid 
Ahora sonreímos, sin duda, pero abiertamente, conscientes de una labor bien hecha, la de tantos estudiosos del Próximo Oriente en España. De alguna manera este artículo pretende ser un acto de gratitud a todos ellos.

\section{Bibliografía}

Borrell Tena, F. et al., 2012, Broadening horizons 3. Conference of Young Researchers working in the Ancient Near East, Barcelona

Córdoba Zoilo, J. M ${ }^{\text {a }}$ (ed.), 1998, Actas del Ier Symposium Internacional. Una década de estudios sobre el Oriente antiguo (1986-1996). Isimu 1.

Córdoba Zoilo, J. Ma (dir.), 2016, En los Confines de Oriente Próximo: El Hallazgo moderno del país de Magán. Madrid.

Córdoba Zoilo, J.Ma'., 2018, Aula didáctica Antonio Blanco Freijeiro. Un Laboratorio para la práctica de la Historia Antigua de Oriente Próximo y Medio. Supplementa ad Isimu. Series I: Studia, Vol. 4, Madrid.

Córdoba Zoilo, J. M y y Pérez Díe, M. C., 2006, La aventura española en oriente (1166-2006): viajeros, museos y estudiosos en la Historia del redescubrimiento del Oriente Próximo antiguo; la arqueología española en Oriente: nacimiento y desarrollo, Vol 2 , Madrid.

Córdoba Zoilo, J. Ma , et al. (Coord.), 2008, Proceedings of the 5th International Congress on the Archaeology of the Ancient Near East, Madrid

Egea Vivancos, A. y Martínez García, J.J., 2017, “¿Orientalismo en Murcia? La labor del Profesor Antonino González Blanco", Estudios Orientales 8, pp. 9-14.

Egea Vivancos, A., et al. (Coords.), 2018, Y la arqueología llegó al aula. La cultura material y el método arqueológico para la enseñanza de la historia y el patrimonio. Gijón.

Feliu, F., et al., 2013, Time and History in the Ancient Near East, Winona Lake.

Gallardo Carrillo, J. y Matilla Séiquer, G., 1997, “Tell Qara Qûzâq. Un excepcional descubrimiento", Revista de Arqueología 198, pp. 24-35.

García Fernández, M., et al., 2013, "Investigación arqueológica en Hirbat alJerahiya (Iraq), noviembre de 2011-noviembre de 2017", Memoria académica 2012-2013, Universidad Pontifica de Comillas, Proyecto I+D.

García Recio, J., 2018, Informe de la visita preliminar a Tell Bismaya (Adab) (sin publicar).

Martín, C., 2007, Las ayudas a proyectos de investigación arqueológica en el exterior de la dirección general de bellas artes y bienes culturales del ministerio de cultura. Instituto del Patrimonio Cultural de España, Madrid.

Martín Galán, R., 2004-2005 "El período helenístico: pervivencia del legado autóctono", Cuadernos del Seminario Water Andrae 7, pp. 23-32.

Matilla Séiquer, G., 2001, Arqueología y antropología en el Alto Éufrates sirio: Tell Jamîs, Murcia.

Vidal, J., 2016, Historia del Instituto del Próximo Oriente Antiguo (1971-2012). Barcelona. 